EPJ Web of Conferences 81, 04009 (2014)

DOI: $10.1051 /$ epjconf/ 20148104009

(C) Owned by the authors, published by EDP Sciences, 2014

\title{
Highlight of Charm Physics at BESIII
}

\author{
P. L. Liu'1,2,3, a \\ for the BESIII Collaboration \\ ${ }^{1}$ Shandong University, Jinan, China \\ ${ }^{2}$ University of Chinese Academy of Sciences, Beijing, China \\ ${ }^{3}$ Institute of High Energy Physics, Beijing, China
}

\begin{abstract}
In this talk, we present the recent results of charm physics from the BESIII collaboration. This talk covers the studies of the leptonic and semi-leptonic decays of $D$ mesons, and the measurement of the strong phase difference based on the quantum correlation.
\end{abstract}

\section{Introduction}

The BESIII [1] experiment at the BEPCII collider started data taking since 2008. For the study of charm meson decays, about $2.9 \mathrm{fb}^{-1}$ data on the $\psi(3770)$ peak has been accumulated. $\psi(3770)$ dominantly decays into $D \bar{D}$, which provides an ideal place for studying the decays of $D^{0}$ and $D^{+}$ mesons.

\section{Determination of $\mathcal{B}\left(D^{+} \rightarrow \mu^{+} v\right)$ and the decay constant $f_{D^{+}}$}

In the Standard Model of particle physics, the $D^{+}$meson can decay into $l^{+} v_{l}$ via a virtual $W^{+}$ boson. The decay rate depends on the $D^{+}$decay constant $f_{D^{+}}$, in which all of the strong interaction effects between the two initial-state quarks are absorbed. The decay width of $D^{+} \rightarrow l^{+} v_{l}$ is given by [2]

$$
\Gamma\left(D^{+} \rightarrow l^{+} v_{l}\right)=\frac{G_{F}^{2} f_{D^{+}}^{2}}{8 \pi}\left|V_{c d}\right|^{2} m_{l}^{2} m_{D^{+}}\left(1-\frac{m_{l}^{2}}{m_{D^{+}}^{2}}\right)^{2},
$$

where $G_{F}$ is the Fermi coupling constant. So by measuring the branching ratio of $D^{+} \rightarrow l^{+} v_{l}$, the decay constant $f_{D^{+}}$can be determined to test the calculations from lattice QCD. In addition, $\left|V_{c d}\right|$ can be directly accessed. $f_{D^{+}}$is also helpful to constrain the CKM matrix element $\left|V_{t d}\right|$ through its relation to the mixing parameter $x_{B}$ and the ratio $f_{D} / f_{B}[3,4]$.

To determine the branching ratio of $D^{+} \rightarrow \mu^{+} v_{\mu}$, the $D$ tagging method is used. Firstly, $D^{-}$mesons are reconstructed with 9 hadronic decay modes $\left(D^{-} \rightarrow K^{+} \pi^{-} \pi^{-}, K_{S}^{0} \pi^{-}, K_{S}^{0} K^{-}, K^{+} K^{-} \pi^{-}, K^{+} \pi^{-} \pi^{-} \pi^{0}\right.$, $\pi^{+} \pi^{-} \pi^{-}, K_{S}^{0} \pi^{-} \pi^{0}, K^{+} \pi^{-} \pi^{-} \pi^{-} \pi^{+}$and $\left.K_{S}^{0} \pi^{-} \pi^{-} \pi^{+}\right)$, and then $\mu^{+}$is sought out in the remaining tracks. $M_{\text {miss }}^{2}=\left(E_{\text {beam }}-E_{D^{-}}-E_{\mu^{+}}\right)^{2}-\left(-\vec{p}_{D^{-}}-\vec{p}_{\mu^{+}}\right)^{2}$ is used to extract the signal events which peak

\footnotetext{
a. e-mail: liupl@ihep.ac.cn
} 
around 0 . Finally, with $2.9 \mathrm{fb}^{-1} \psi(3770)$ data, the branching fraction of $D^{+} \rightarrow \mu^{+} v_{\mu}$ is measured to be [5]

$$
\mathcal{B}\left(D^{+} \rightarrow \mu^{+} v_{\mu}\right)=(3.71 \pm 0.19 \pm 0.06) \times 10^{-4} .
$$

With combination of $G_{F}$, the mass of $\mu^{+}$and $D^{+}$, the lifetime of $D^{+}$and $\left|V_{c d}\right|$ from the global fit in the SM [6], the decay constant $f_{D^{+}}$is obtained with the best precision in the world [5]

$$
f_{D^{+}}=(203.2 \pm 5.3 \pm 1.8) \mathrm{MeV}
$$

\section{Measurement of $\mathcal{B}\left(D^{0} \rightarrow K^{-} / \pi^{-} e^{+} v\right)$ and the Form-Factor $f_{+}\left(q^{2}\right)$}

The differential decay rate of $D^{0} \rightarrow K^{-}\left(\pi^{-}\right) e^{+} v$ is written as

$$
\frac{\Delta \Gamma(D \rightarrow K(\pi) e v)}{d q^{2}}=\frac{G_{F}^{2}\left|V_{c s(d)}\right|^{2}}{24 \pi^{3}} p^{3}\left|f_{+}\left(q^{2}\right)\right|^{2} .
$$

With the precisely determined $V_{c s(d)}$ from the unitarity of the CKM matrix, we can obtain the formfactor $f_{+}\left(q^{2}\right)$ to check the lattice QCD calculations.

This analysis has been done using one-third of the $2.9 \mathrm{fb}^{-1} \psi(3770)$ data, with a partialblind method. Firstly, the singly tagged $\bar{D}^{0}$ is reconstructed with four hadronic modes $\left(\bar{D}^{0} \rightarrow\right.$ $K^{+} \pi^{-}, K^{+} \pi^{-} \pi^{0}, K^{+} \pi^{-} \pi^{0} \pi^{0}$ and $\left.K^{+} \pi^{-} \pi^{-} \pi^{+}\right)$. The signal candidates are searched by reconstructing two opposite charged tracks $\left(K^{+} / \pi^{+} e^{-}\right)$left in the event, and $U_{\text {miss }}=E_{\text {miss }}-p_{\text {miss }}$ is used to extract the signal events which peak around 0 . Here, $E_{\text {miss }}=E_{\text {beam }}-E_{\text {hadron }}-E_{\text {electron }}$, and $p_{\text {miss }}=\left|-\vec{p}_{\text {hadron }}-\vec{p}_{\text {electron }}\right|$. The branching fractions are measured to be

$$
\begin{aligned}
& \mathcal{B}\left(D^{0} \rightarrow K^{-} e^{+} v\right)=(3.542 \pm 0.030 \pm 0.067) \% \\
& \mathcal{B}\left(D^{0} \rightarrow \pi^{-} e^{+} v\right)=(0.288 \pm 0.008 \pm 0.005) \% .
\end{aligned}
$$

These two results are consistent with the world averages [6].

To measure the form factor, the partial decay rates are measured in different $q^{2}$ bins, where $q^{2}$ is the squared invariant mass of $e^{+} v$ system. The $f_{+}\left(q^{2}\right)$ distributions are shown in figure 1 with theoretical curves overlaid [7].
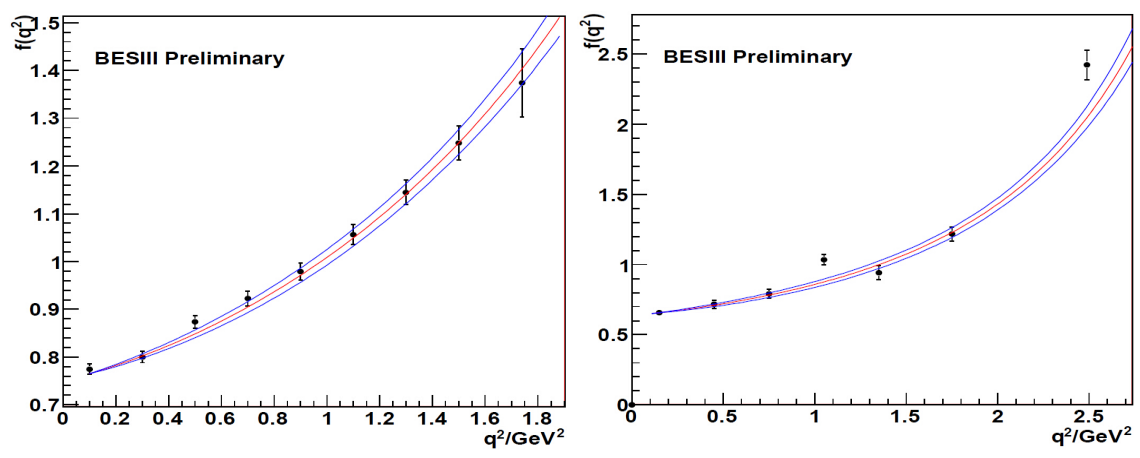

Figure 1. $f_{+}\left(q^{2}\right)$ distributions for the decay of $D^{0} \rightarrow K^{-} e^{+} v$ (left) and $D^{0} \rightarrow \pi^{-} e^{+} v$ (right). Points are measured from data, curves are the theoretical predictions varying within one statistical standard sigma. 
MESON 2014-13 ${ }^{\text {th }}$ International Workshop on Production, Properties and Interaction of Mesons

\section{Measurement of the strong phase difference $\delta_{K \pi}$}

Studying the $D^{0}-\bar{D}^{0}$ mixing is important for identifying the size of the long distance effect and searching for new physics [8], and also helpful for studying CP violation in charm physics. Charm mixing is described by two dimensionless parameters $x$ and $y$. The measurement of the strong phase difference $\delta$ between the doubly Cabibbo-suppressed (DCS) decay $\overline{D^{0}} \rightarrow K^{-} \pi^{+}$and the corresponding Cabibbo-favored (CF) $D^{0} \rightarrow K^{-} \pi^{+}$, allows $x$ and $y$ to be extracted from $x^{\prime} \equiv x \cos \delta+y \sin \delta$ and $y^{\prime} \equiv y \cos \delta-x \sin \delta$ [9]. Furthermore, finer precision of $\delta$ helps the $\gamma / \phi_{3}$ angle measurement in CKM matrix according to the so-called ADS method [6].

Using the quantum-correlated technique, $\delta$ can be accessed using the following formula

$$
2 r \cos \delta+y=\left(1+R_{\mathrm{WS}}\right) \cdot \mathcal{A}_{C P \rightarrow K \pi},
$$

where $\mathcal{A}_{C P \rightarrow K \pi}$ is the asymmetry between the branching fractions of CP-odd and CP-even states decaying to $K^{-} \pi^{+} \cdot \mathcal{B}\left(D^{C P^{ \pm}} \rightarrow K^{-} \pi^{+}\right)$are measured with $D$ tagging method using 5 CP-even $D^{0}$ decay modes $\left(K^{+} K^{-}, \pi^{+} \pi^{-}, K_{S}^{0} \pi^{0} \pi^{0}, \pi^{0} \pi^{0}\right.$ and $\left.\rho^{0} \pi^{0}\right)$ and 3 CP-odd modes $\left(K_{S}^{0} \pi^{0}, K_{S}^{0} \eta\right.$ and $\left.K_{S}^{0} \omega\right)$. With $2.9 \mathrm{fb}^{-1} \psi(3770)$ data, we obtain the asymmetry to be [10]

$$
\mathcal{A}_{C P \rightarrow K \pi}=(12.7 \pm 1.3 \pm 0.7) \% \text {. }
$$

By quoting the external inputs of $R_{\mathrm{D}}=r^{2}=(3.50 \pm 0.04) \%$ o $y=(6.7 \pm 0.9) \%$ from HFAG 2013 [11] and $R_{\mathrm{WS}}=(3.80 \pm 0.05) \%$ from PDG [6], we obtain [10]

$$
\cos \delta_{K \pi}=1.02 \pm 0.11 \pm 0.06 \pm 0.01
$$

where the third uncertainty is due to the errors introduced by the external input parameters. This result provides the world best constrain to $\delta_{K \pi}$.

\section{Measurement of the Relative Strong-phase Difference between $D^{0}$ and $\bar{D}^{0}$ decay to $K_{S}^{0} \pi^{+} \pi^{-}$}

The CKM angle $\gamma / \phi 3$ can be measured from the decay $B^{+} \rightarrow D\left(K_{S}^{0} \pi^{+} \pi^{-}\right) K^{ \pm}$through the GGSZ method $[12,13]$. The Dalitz plot can be separated into $2 N$ bins as shown in figure 2(a). The $N$ bins lying below the symmetry axis are denoted by the index $i$, while the remaining bins are indexed with $\bar{i}$, and these square bins are symmetric under exchange of $x$ and $y\left(x \equiv m_{K_{s}^{0} \pi^{-}}^{2}, y \equiv m_{K_{s}^{0} \pi^{+}}^{2}\right)$. The two parameters $c_{i}$ and $s_{i}$ denote the weighted average of $\cos \left(\Delta \delta_{D}\right)$ and $\sin \left(\Delta \delta_{D}\right)$ in the $i^{\text {th }}$ bin, respectively. And $\Delta \delta_{D}$ is the strong phase difference between the DCS decay $\bar{D}^{0} \rightarrow K_{S}^{0} \pi^{+} \pi^{-}$and the corresponding CF decay $D^{0} \rightarrow K_{S}^{0} \pi^{+} \pi^{-}$.

The number of events in the $i^{\text {th }}$ bin of a Dalitz plot originating from the $B^{ \pm}$decay is

$$
N_{i}^{ \pm}=\frac{a_{B}}{a_{D}}\left[K_{i}+r_{B}^{2} K_{\bar{i}} \pm 2 r_{B} \sqrt{K_{i} K_{\bar{i}}}\left(\cos \left(\delta_{\mathrm{B}}+\gamma\right) \mathrm{c}_{\mathrm{i}}+\sin \left(\delta_{\mathrm{B}}+\gamma\right) \mathrm{s}_{\mathrm{i}}\right)\right] .
$$

The normalization factors, $r_{B}$ and $\delta_{B}$ could be determined at $B$ factories. Using $\psi(3770), K_{i}$ can be obtained by flavor tagged $D \rightarrow K_{S}^{0} \pi^{+} \pi^{-}$decays, $c_{i}$ can be obtained from CP tagged $D \rightarrow K_{S}^{0} \pi^{+} \pi^{-}$ decays, and $s_{i}$ is obtained from double Dalitz decays $D^{0} \rightarrow K_{S}^{0} \pi^{+} \pi^{-}$and $\overline{D^{0}} \rightarrow K_{S}^{0} \pi^{+} \pi^{-}$.

The Dalitz plot is split into 8 bins as shown in figure 2(b), which have been optimized, to get the highest sensitivity of $\gamma$. The results of $c_{i}$ and $s_{i}$ in each bin are given in figure 2(c). Our results will allow for increased precision in the measurement of $\gamma / \phi 3$. 


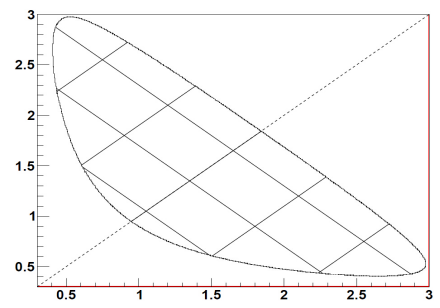

(a)

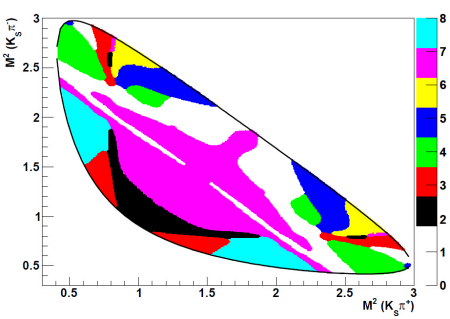

(b)

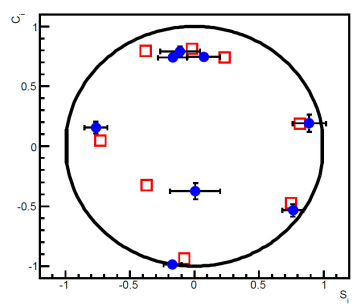

(c)

Figure 2. (a): Squared binned Dalitz plot with symmetric bins over an exchange of $x$ and $y$. (b): Modified optimal binning. (c): Red squares mark the model predicted value of $c_{i}$ and $s_{i}$ [14], and the blue dot mark fitted value from data with statistical error.

\section{Summary}

Based on the $2.9 \mathrm{fb}^{-1}$ data taken at the $\psi(3770)$ peak, the recent results on the study of charm physics are presented. The BESIII experiment aims at getting $20 \mathrm{fb}^{-1} \psi(3770)$, and more promising results are expected.

\section{Acknowledgements}

We thank the staff of BEPCII and the computing center for their strong support, and our BESIII collaborators for their efforts in physics.

\section{References}

[1] M. Ablikim et al. [BESIII Collaboration], Phys. Rev. Lett. 110, 252001 (2013)

[2] D. Silverman and H. Yao, Phys. Rev. D 38, 214 (1988)

[3] C. Bernard et al., arXiv:hep-ph/9709328

[4] K. Hara et al. [Belle Collaboration], Phys. Rev. Lett. 110, 131801 (2013)

[5] M. Ablikim et al. [BESIII Collaboration], Phys. Rev. D 89, 051104 (2014)

[6] J. Beringer et al. [Particle Data Group], Phys. Rev. D 86, 010001 (2012)

[7] John A. Bailey et al., arXiv:1111.5471

[8] S. Bianco, F. L. Fabbri, D. Benson and I. Bigi, Riv. Nuovo Cim. 26N7, 1 (2003)

[9] R. Aaij et al. [LHCb Collaboration], Phys. Rev. Lett. 110, 101802 (2013)

[10] M. Ablikim et al. [BESIII Collaboration], Phys. Lett. B 734, 227(2014)

[11] Heavy Flavor Averaging Group: http://www.slac.stanford.edu/xorg/hfag/charm/

[12] A. Giri, Y. Grossman, A. Soffer, and J. Zupan, Phys. Rev. D 68, 054018 (2003)

[13] A. Bondar, A. Poluektov, Eur. Phys.J.C 47, 347 (2006)

[14] B. Aubert et al. [BABAR Collaboration], Phys. Rev. D 78, 034023 (2008) 